\title{
Incidence and predictors of left atrial thrombus development in patients scheduled for repeat catheter ablation for atrial fibrillation: Is transesophageal echocardiography always mandatory?
}

\author{
Satoshi Yanagisawa ${ }^{1}$, Yasuya Inden ${ }^{2}$, Shuro Riku², Kazumasa Suga ${ }^{2}$, Koichi Furui², \\ Toshifumi Nakagomi ${ }^{2}$, Masafumi Shimojo ${ }^{3}$, Takashi Okajima ${ }^{3}$, Rei Shibata ${ }^{1}$, and Toyoaki \\ Murohara $^{3}$ \\ ${ }^{1}$ Nagoya University Graduate School of Medicine \\ ${ }^{2}$ Nagoya University \\ ${ }^{3}$ Nagoya University Hospital
}

March 5, 2021

\begin{abstract}
Introduction: The risk of developing left atrial (LA) thrombi after initial catheter ablation for atrial fibrillation (AF) and requirements for imaging evaluation for thrombi screening at repeat ablation is unclear. This study aimed to assess the occurrence of thrombus development and frequency of any imaging study evaluating thrombus formation during repeat ablation for AF. Methods: Of 2,066 patients undergoing initial catheter ablation for AF with uninterrupted oral anticoagulation, 615 patients underwent repeat ablation after 258.0 (105.0-882.0) days. We investigated which factors were associated with safety outcomes and requirements for thrombi screening. Results: All patients underwent at least one imaging examination to screen for thrombi in the first session, but the examination rate decreased to 476 patients $(77 \%)$ before the repeat procedure. The frequency of imaging evaluations was $5.0 \%, 11 \%, 21 \%, 84 \%$, and $91 \%$ for transesophageal echocardiography and $18 \%, 33 \%, 49 \%$, $98 \%$, and $99 \%$ for any imaging modality at repeat ablation performed [?]60 days, [?]90 days, [?]180 days, $>180$ days, and $>1$ year after the initial procedure, respectively. Three patients $(0.5 \%)$ developed LA thrombi at repeat ablation due to identifiable causes, and no patients had thromboembolic events when no imaging evaluation was performed. Multivariate analysis revealed that repeat ablation $>180$ days, non-paroxysmal atrial arrhythmias, and lower left ventricular ejection fraction were predictors of the risk of thrombus development. Conclusions: The risk development of thrombus at repeat ablation for AF was low. There needs to be a risk stratification for the requirement of imaging screening for thrombi at repeat ablation for AF.
\end{abstract}

Incidence and predictors of left atrial thrombus development in patients scheduled for repeat catheter ablation for atrial fibrillation: Is transesophageal echocardiography always mandatory?

Satoshi Yanagisawa, MD, PhD. ${ }^{1,2^{*}}$; Yasuya Inden, MD, PhD. ${ }^{2}$; Shuro Riku, M.D. ${ }^{2}$; Kazumasa Suga, M.D. ${ }^{2}$; Koichi Furui, M.D. ${ }^{2}$; Toshifumi Nakagomi, M.D. ${ }^{2}$; Masafumi Shimojo, M.D. ${ }^{2}$; Takashi Okajima M.D. ${ }^{2}$; Rei Shibata, MD, PhD. ${ }^{1}$; and Toyoaki Murohara, MD, PhD. ${ }^{2}$

${ }^{1}$ Department of Advanced Cardiovascular Therapeutics, Nagoya University Graduate School of Medicine, Nagoya, Japan

${ }^{2}$ Department of Cardiology, Nagoya University Graduate School of Medicine, Nagoya, Japan

*Address for correspondence :

Satoshi Yanagisawa, MD, PhD 
Department of Advanced Cardiovascular Therapeutics

Nagoya University Graduate School of Medicine

65 Tsurumai-cho, Showa-ku, Nagoya, Aichi, 466-8550, Japan.

Tel.: +81-52-744-2147

Fax: $+81-52-744-2138$

E-mail address: pinponstar@yahoo.co.jp

Running title : Evaluation of LA thrombi at repeat ablation

Disclosures: Drs. Yanagisawa and Shibata are affiliated with a department sponsored by Medtronic Japan. Other authors have no conflict of interest.

Funding : none

\section{Abstract}

Introduction: The risk of developing left atrial (LA) thrombi after initial catheter ablation for atrial fibrillation $(\mathrm{AF})$ and requirements for imaging evaluation for thrombi screening at repeat ablation is unclear. This study aimed to assess the occurrence of thrombus development and frequency of any imaging study evaluating thrombus formation during repeat ablation for AF.

Methods: Of 2,066 patients undergoing initial catheter ablation for AF with uninterrupted oral anticoagulation, 615 patients underwent repeat ablation after 258.0 (105.0-882.0) days. We investigated which factors were associated with safety outcomes and requirements for thrombi screening.

Results: All patients underwent at least one imaging examination to screen for thrombi in the first session, but the examination rate decreased to 476 patients $(77 \%)$ before the repeat procedure. The frequency of imaging evaluations was $5.0 \%, 11 \%, 21 \%, 84 \%$, and $91 \%$ for transesophageal echocardiography and $18 \%$, $33 \%, 49 \%, 98 \%$, and $99 \%$ for any imaging modality at repeat ablation performed [?]60 days, [?] 90 days, [?]180 days, $>180$ days, and $>1$ year after the initial procedure, respectively. Three patients $(0.5 \%)$ developed LA thrombi at repeat ablation due to identifiable causes, and no patients had thromboembolic events when no imaging evaluation was performed. Multivariate analysis revealed that repeat ablation $>180$ days, nonparoxysmal atrial arrhythmias, and lower left ventricular ejection fraction were predictors of the risk of thrombus development.

Conclusions: The risk development of thrombus at repeat ablation for AF was low. There needs to be a risk stratification for the requirement of imaging screening for thrombi at repeat ablation for $\mathrm{AF}$.

Keywords: catheter ablation; atrial fibrillation; repeat session; transesophageal echocardiography; thrombus.

\section{Abbreviations}

$\mathrm{AF}=$ atrial fibrillation

$\mathrm{CT}=$ computed tomography

$\mathrm{DOAC}=$ direct oral anticoagulants

$\mathrm{LA}=$ left atrium

LAA $=$ left atrial appendage

$\mathrm{LAD}=$ left atrial diameter

$\mathrm{LVEF}=$ left ventricular ejection fraction

$\mathrm{PV}=$ pulmonary vein 
$\mathrm{SEC}=$ spontaneous echocardiography contrast

TEE $=$ transesophageal echocardiography

\section{Introduction}

Catheter ablation is an effective treatment for suppressing atrial fibrillation (AF) and has a high success rate. $^{1,2}$ With the recent development of ablation technology and mapping devices and growing indications of catheter ablation in patients with $\mathrm{AF}$, most patients are recurrence-free from $\mathrm{AF}$ after the initial catheter ablation procedure. However, the success rate is not absolute, and some patients have a recurrence after the initial ablation. These patients are often scheduled to undergo repeat sessions for recurrent AF when associated with intolerable symptoms and frequent occurrence.

Transesophageal echocardiography (TEE) is the gold standard for evaluating left atrial (LA) thrombi before $\mathrm{AF}$ ablation, and a recent expert consensus report recommended the necessity of excluding the possibility of LA thrombi before the initial AF catheter ablation. ${ }^{3}$ Nonetheless, the relevance of evaluating the presence of thrombi at repeat sessions due to recurrence remains unclear. After the first session, although patients have a recurrence, the burden and duration of AF may significantly decrease, and these patients usually continue oral anticoagulants following the procedure, which could indicate that the thrombi are unlikely to develop in those patients after the first session. ${ }^{4}$ It is unclear whether imaging examination to screen for LA thrombi is necessary at repeat sessions, especially in patients at low risk and in early repeat ablation. Since TEE is associated with the risk of aspiration pneumonia and pharyngeal perforation, although with a very low prevalence, it is important to avoid unnecessary examinations which impose a burden on the patient and cause discomfort before the repeat ablation.

To test this hypothesis, the present study was conducted to assess the occurrence of thrombi at repeat ablation for AF recurrence after the first session and the frequency of any imaging study, including TEE and contrast-enhanced computed tomography (CT) imaging, for thrombi screening before the repeat procedure in a large cohort study. We investigated which factors and time to repeat ablation were associated with safety outcomes regarding thrombi development during AF repeat ablation and with the requirement for invasive imaging studies for evaluating thrombi development.

\section{Methods}

\section{Study population}

The study population was retrospectively analyzed and originated from a database of patients who underwent catheter ablation for AF at Nagoya University Hospital, Japan. All patients who were scheduled to undergo catheter ablation for AF which involved the LA at our center from January 2007 to July 2021 were included in the study. Patients with a history of MAZE procedure and thrombi formation prior to the initial session and with major adverse events during the first session were also included in the study. The exclusion criteria were as follows: detection of thrombi at the first session; and undergoing ablation for the right atrium only. We reviewed the medical records, examination data, and procedures in the databases of all eligible patients. The indications for catheter ablation complied with the most recent guidelines. ${ }^{3}$ Antiarrhythmic agents were discontinued at least five half-lives before ablation. Informed consent for the procedure was obtained from all patients. The study protocol was approved by the institutional ethics committee. The study was performed in accordance with the principles of the Declaration of Helsinki.

Periprocedural examination and anticoagulant management

Patients scheduled for catheter ablation were admitted the day before the procedure. At admission, baseline blood testing, echocardiography, and electrocardiography were performed. All patients underwent standard transthoracic two-dimensional echocardiography before the procedure. Left ventricular ejection fraction (LVEF) was analyzed using the biplane Simpson's technique. Left atrial diameter (LAD) was assessed using the M-mode method. For the coagulation marker assessment, blood samples for each patient were collected the day before ablation, as described previously. ${ }^{5}$ 
The patients received oral anticoagulants at least 3-4 weeks before the ablation procedure. The attending physician chose direct oral anticoagulants (DOACs) or warfarin. Each DOAC was prescribed per the drug package insert (apixaban: 5 or $2.5 \mathrm{mg}$ twice daily; dabigatran: 150 or $110 \mathrm{mg}$ twice daily; rivaroxaban: 15 or $10 \mathrm{mg}$ once daily; and edoxaban: 60 or $30 \mathrm{mg}$ once daily). The warfarin dose was adjusted to maintain a therapeutic international normalized ratio of 2.0-3.0 (or 1.6-2.6 for patients [?]70 years old), according to guidelines. ${ }^{6}$ The aforementioned anticoagulants were uninterruptedly administered throughout the procedure. ${ }^{7}$

\section{Catheter ablation procedures}

The ablation procedure was performed using a minimal sedation strategy while keeping the patient conscious. An 80-100 IU/kg bolus of heparin was administered immediately after sheath insertion, and the target range for an activated clotting time of 300-350 s was maintained using additional heparin infusions.

This study included four catheter ablation techniques (radiofrequency, cryoballoon, hot balloon, and laser balloon ablation). Radiofrequency and cryoballoon ablation were performed in most cases. The radiofrequency ablation method was described previously. ${ }^{7}$ Two 8 -Fr sheaths and an 8.5-Fr steerable sheath were introduced into the LA through a trans-septal puncture using intracardiac echocardiography (ICE). The electrical pulmonary vein (PV) isolation was performed using a circular mapping catheter placed on the ostium of the PV. The ablation procedures were performed using a 3D mapping system. We have used a 3.5-mm-tip open-irrigated ablation catheter and contact force-sensing ablation catheter since 2009 and 2013, respectively. The radiofrequency energy output was adjusted to $20-35 \mathrm{~W}$ at a flow rate of $8-30 \mathrm{~mL} / \mathrm{min}$, with a maximum temperature of $42^{\circ} \mathrm{C}$. The contact force was targeted as 10-20 g, and the maximum contact force threshold was set to $50 \mathrm{~g}$. In most patients with paroxysmal and early persistent AF, we only performed PV isolation and cavotricuspid isthmus ablation. However, additional linear and substrate ablations were applied in patients with atrial flutter or long-term persistent AF. If sinus rhythm was not achieved by the end of the ablation, external cardioversion was performed.

For the cryoballoon ablation procedure, a 12-Fr steerable sheath was inserted into the LA. A secondgeneration 28-mm cryoballoon system (Arctic Front Advance, Medtronic) was advanced into the ostium of each PV using an inner circular mapping catheter. After confirming the PV occlusion with the cryoballoon, a 120-180-s cycle freeze ablation was repeated until electrical isolation of the PV was achieved. Other hot balloon and laser balloon ablations were performed according to the standard approach as per the recommendation of the manufacturer and expert consensus reports. ${ }^{3}$

TEE and contrast-enhanced CT examination

TEE was performed within 24 hours of ablation. After local pharyngeal anesthesia, the transducer was inserted into the midportion of the esophagus. We assessed the presence of intra-LA thrombus, left atrial appendage (LAA) orifice area, LAA flow velocity, spontaneous echocardiography contrast (SEC), and LA-right atrium shunt using a multiplane transesophageal transducer connected to an ultrasound system (Vivid E95, GE Vingmed Ultrasound AS, Horten, Norway). The LAA flow velocity was assessed using the pulsed Doppler method, with the sample volume placed within the LAA cavity. The peak LAA in- and outflow emptying velocities were analyzed for at least five cardiac cycles, and mean values were calculated. A thrombus was defined as an echo-dense mass adherent to the cavity showing independent motion or different echogenicity that could be distinguished from the surrounding endocardium or pectinate muscles observed in multiple planes. The SEC was defined as dynamic, "smoke like" swirling patterns of echogenicity in the LA and LAA distinct from white-noise artifacts after gain setting adjustment to eliminate background noise. The SEC severity was divided into 5 grades (0: none to 4: severe) according to the echo density and the extent of SEC in the LA and LAA cavities. ${ }^{8}$ The absence/presence of thrombi and SEC were confirmed by at least two investigators who were blinded to the baseline characteristics of the patients and related outcomes.

Contrast-enhanced CT imaging was also scheduled before the procedure in all cases if applicable. Although the CT image was primarily used for spatial reorganization of the 3D image of the LA during the mapping procedure, it is also useful to evaluate the absence of the LA and LAA thrombi in a delayed contrast image 
since it has a high negative predictive value. ${ }^{9}$ We offered both TEE and contrast-enhanced CT imaging for most patients undergoing first catheter ablation for AF; however, some patients who could not be examined by TEE for any reason underwent CT imaging study only to confirm the absence of thrombi. In contrast, when thrombi were suspected on the CT image, a final decision for the presence of thrombi was made using TEE in all cases. If patients could not be examined by both TEE or CT imaging, ICE was used to evaluate thrombi during the ablation procedure. The presence or absence of LA thrombi on all CT images was reviewed and diagnosed by a radiologist who was blinded to the patient characteristics.

Outcome measurement after the first session

After the initial session, patients were followed-up at an outpatient clinic in our institution and a nearby practitioner every month at minimum thereafter. At each follow-up, electrocardiography and 24-h Holter testing were performed, if necessary. Recurrence was defined as an atrial arrhythmia lasting [?]30 s in examination tests after a blanking period of 3 months after ablation regardless of the use of anti-arrhythmic drugs. Repeat ablation was considered when patients had a recurrence of AF with intolerable symptoms, frequent occurrence, or refractoriness to anti-arrhythmic drugs. However, some patients underwent repeat sessions within the blanking period due to the recurrence of $\mathrm{AF}$, which was left at the discretion of the attending physician.

Oral anticoagulants were continued for at least three months after the first session. During the follow-up, we discontinued oral anticoagulant administration when the patients were free from AF recurrence and had a low risk of comorbidities, as defined by a low $\mathrm{CHADS}_{2}$ score and absence of history of stroke. Any related outcomes after discharge, including heart failure or AF hospitalization, pacemaker implantation, cardiovascular intervention, and cardioversion were assessed after the first session in all patients undergoing repeat sessions as found in the patient medical records.

Repeat ablation

Patients scheduled for repeat ablation were admitted the day before the procedure. Blood, electrocardiography, and echocardiography testing were performed in the same manner as in the initial session. Updated information on oral anticoagulant use and baseline characteristics just before the repeat procedure was obtained from the medical records. TEE was also performed within 24 hours of the repeat ablation. Transthoracic echocardiography and contrast-enhanced CT images were generally examined within a week before ablation. Although the decision about the type of imaging examination performed, including TEE and/or CT imaging, was made depending on the patients' condition and was left to the discretion of the attending physician, both TEE and CT examinations were planned in most patients, if applicable. The final confirmation of thrombi presence was assessed by TEE examination in cases with a possibility of presence of thrombi in any imaging study, as performed in the first session.

Statistical analysis

Continuous variables were expressed as mean +- standard deviation or median (interquartile range). Differences in baseline characteristics were analyzed using the Student's $t$-test for parametric data and the Mann-Whitney $U$ test for non-parametric data. Categorical variables were compared using chi-square or Fisher's exact tests. The prognostic value of each factor was first evaluated using a univariate logistic regression analysis. Factors with p-values $<0.05$ in the univariate analysis were entered into a multivariate logistic regression model using a forward stepwise method to identify independent predictors. Statistical significance was set at $\mathrm{p}<0.05$.

\section{Results}

Baseline patient characteristics

A total of 2,066 eligible patients were included in the study. Radiofrequency, cryoballoon, hot balloon, and laser balloon ablations were applied in 1,589, 412, 51, and 11 patients, respectively. A total of 801 (39\%) patients showed AF recurrence during a mean follow-up period of 14.3 months. Repeat ablation 
was performed in 615 patients 258.0 (105.0-882.0) days after the first session. Baseline characteristics and examination data for the total sample and patients with and without repeat ablation are shown in Table 1. Of the patients who underwent repeat ablation, the mean age was $62.7+-10.8$ years and $75 \%$ were male. More than half of the patients $(63 \%)$ had paroxysmal AF. Two hundred and ninety-four patients $(48 \%)$ received DOACs and 12 patients had a history of thrombus detection prior to the first ablation, followed by elimination of the thrombi at the time of the first session. In the first session, 598 patients (97\%) and 576 (94\%) underwent TEE and contrast-enhanced CT examination, respectively. All patients underwent at least one imaging examination to screen for the presence of thrombi at the time of the first ablation.

Repeat ablation

At repeat ablation, $\mathrm{CHADS}_{2}$ and $\mathrm{CHA}_{2} \mathrm{DS}_{2}$-VASc scores increased significantly and there was an improvement in LVEF (Table 2). TEE and contrast-enhanced CT imaging were performed in 356 (58\%) and 408 patients $(66 \%)$, respectively. Twenty-three patients refused to undergo TEE at the repeat ablation, mostly due to the difficulty in swallowing the probe, but all patients underwent some alternative imaging examination. A total of 476 patients (77\%) underwent at least one imaging modality to evaluate LA thrombi before the repeat procedure. One hundred and eighteen patients $(19 \%)$ had an interruption of oral anticoagulants before the repeat session, and 142 patients (23\%) exhibited a non-paroxysmal atrial arrhythmia after the first ablation. Changes in coagulation markers and TEE findings from the first to the repeat ablation are shown in Table 3. At the repeat ablation, 72 patients switched from warfarin to DOAC, while six patients switched from DOAC to warfarin. Three patients ( $0.8 \%$ in TEE and $0.5 \%$ in repeat ablation) developed LA thrombi seen on TEE at the repeat procedure. In contrast, one patient had a thromboembolic event after the repeat ablation procedure without prior TEE evaluation.

Details of patients who developed LA thrombi at repeat ablation

The time to repeat ablation in the three patients who developed thrombi was $1,449,515$, and 224 days after the initial session (Table 4). All patients underwent TEE before the first session. Patient 1 was a 44year-old man with paroxysmal AF. He had a recurrence 6 months after the first session, but antiarrhythmic drug administration was able to suppress the AF occurrence thereafter. Anticoagulation, warfarin was discontinued with reference to the low-risk profile of this patient $\left(\mathrm{CHADS}_{2}\right.$ score 0$)$. Three years later, he returned to our hospital because of the frequent occurrence of $\mathrm{AF}$ and underwent a repeat session. Apixaban was administered alternatively prior to the procedure. TEE showed a thrombus attached to the LAA wall with a significant grade 2 SEC. Patient 2 was a 38 -year-old man with persistent AF. He had a history of heart failure with reduced LVEF $33 \%$ before the first session and a history of LA thrombus prior to the initial session. After successful first ablation, sinus rhythm was maintained, with a remarkable improvement in LVEF. However, atrial flutter with a high-rate ventricular response occurred after 1 year, which could not be controlled by anti-arrhythmic drugs, and required repeat ablation. Echocardiography showed a severely impaired LVEF of $19 \%$ and dilated LAD of $40.7 \mathrm{~mm}$, which might be suggestive of tachycardia-induced cardiomyopathy. Although the patient continued taking dabigatran from the first session onwards, the TEE performed before the repeat ablation revealed LAA thrombi with SEC grade 3. The patient had already been examined by contrast-enhanced CT prior to the repeat ablation and the absence of LA thrombi was confirmed, but the examination time was 29 days before the procedure. We speculated that the severely decreased LVEF due to atrial flutter and heart failure may be a possible explanation for the development of thrombi in this case. Patient 3 was a 62-year-old man with persistent AF. He underwent extensive LA ablation in addition to PV isolation with administration of apixaban $10 \mathrm{mg}$ at the initial session; however, AF recurred after 3 months. Before the repeat ablation, he experienced an intestinal hemorrhage event causing hospitalization at another facility, and the attending doctor reduced the dose of apixaban to $5 \mathrm{mg}$, which was an inappropriate reduced dose, although recurrence AF persisted. The inappropriately reduced dose in this case may be a possible reason for the LAA thrombi with a dense grade 2 SEC. All three patients had no neurological function abnormalities and no thromboembolic events occurred thereafter.

Details of the patient who developed a thromboembolic event without TEE at repeat ablation 
A 66-year-old man taking warfarin underwent radiofrequency ablation for persistent AF. Repeat ablation was planned 85 days after the first session due to the frequent occurrence of intermittent AF. The CHADS 2 and $\mathrm{CHA}_{2} \mathrm{DS}_{2}$-VASc scores were 2 and 3, and LVEF and LAD were $57 \%$ and $50.6 \mathrm{~mm}$, respectively. Warfarin was continued after the first session and was uninterruptedly administered in the repeat session. The patient did not undergo TEE, but contrast-enhanced CT was performed the day before the procedure and the absence of thrombi in the LA and LAA was confirmed. Three days after the ablation, the patient complained of dysarthria and vomiting. Head magnetic resonance imaging demonstrated an acute infarction of the basilar artery. The prothrombin international normalized ratio was 2.23 the day before the procedure, but it decreased to 1.34 at the time of stroke. Subsequent emergency angiography and successful catheter thrombectomy resulted in full recovery. In this case, we concluded that the thrombi which caused the stroke may have developed after the ablation procedure. Extensive radiofrequency ablation under the postprocedure therapeutic range of warfarin may have been the cause of the event.

Evaluation of LA thrombi and time to the repeat ablation

The absence or presence of TEE evaluation and the time to repeat ablation in each patient are shown in Figure 1. Interestingly, the prevalence of TEE examination became predominant after 180 days to repeat ablation. Most patients underwent TEE examination at repeat ablation when performed more than 1 year after the first session; however, some patients refused to undergo the examination because of difficulty in swallowing the probe (this was mostly their experience in the first session). Figure 2 demonstrates the evaluation of thrombi by each imaging modality and the time to repeat ablation in each patient. All but one patient received at least one imaging modality to exclude thrombi in repeat ablation at $>1$-year. The remaining patient was a 57-year-old man with paroxysmal AF. Given the low risk of this patient $\left(\mathrm{CHADS}_{2}\right.$ score of 0) and continuous administration of DOAC after the initial ablation, imaging evaluation prior to the repeat ablation was precluded. The patient underwent successful repeat ablation without adverse events.

The evaluation of LA thrombi based on the specific time to repeat ablation is shown in Figure 3 and Table 5. The rates of TEE and any imaging modality evaluation were $5.0 \%, 11 \%, 21 \%, 84 \%$, and $91 \%$; and $18 \%$, $33 \%, 49 \%, 98 \%$, and $99 \%$ for a time to repeat ablation of [?] 60 days, [?]90 days, [?]180 days, $>180$ days, and $>1$ year, respectively. All three cases of thrombi had been scheduled for repeat ablation $>180$ days after the first procedure. None of the patients who were not evaluated by any imaging modality prior to the repeat session had thromboembolic events.

Figure 4 shows an evaluation of the clinical factors associated with undergoing TEE and any imaging modality during repeat ablation. Patients with non-paroxysmal AF were significantly more likely to undergo TEE and any imaging modality evaluation than those with paroxysmal AF (TEE, $85 \%$ vs. $73 \%, \mathrm{p}=0.001$; any imaging, $70 \%$ vs. $51 \%, \mathrm{p}<0.001)$. Moreover, a history of thrombus detection before the first ablation (TEE, $100 \%$ vs. $57 \%, \mathrm{p}=0.005$ ) and time to repeat ablation $>180$ days (TEE, $84 \%$ vs. $21 \%, \mathrm{p}<0.001$; any imaging, $98 \%$ vs. $49 \%, \mathrm{p}<0.001$ ) were significant factors for suggesting TEE and imaging evaluation at the repeat ablation.

The sample was divided into patients with a time repeat ablation [?]180 days and $>180$ days, and we compared the examination data between these two groups (Table 6). Changes in $\mathrm{CHADS}_{2}$ and $\mathrm{CHA}_{2} \mathrm{DS}_{2^{-}}$ VASc scores between the first and repeat sessions were more evident in the late ablation group than in the early repeat ablation group. The incidence of clinical events after the first session was significantly higher in the late repeat ablation group than in the early ablation group $(26 \%$ vs. $18 \%, \mathrm{p}=0.002)$. Although cardioversion for atrial arrhythmia was the most frequent event, other events also occurred frequently in the late ablation group $(9.2 \%$ vs. $3.1 \%, \mathrm{p}=0.003)$.

Changes in SEC grade on TEE from the first to repeat ablation

Changes in the SEC grade from the first session to the repeat session in patients undergoing TEE are shown in Table 7 and Figure 5. Among patients with a SEC grade 0 in the first session, $46 \%(225 / 493)$ did not undergo TEE at the repeat sessions. This trend was stronger in the early repeat group ([?]180 days) than in the late repeat ablation group (>180 days) $(82 \%$ vs. $16 \%, \mathrm{p}<0.001)$. In contrast, all 11 patients with 
SEC grade 4 at the first session were examined by TEE at repeat sessions, but the SEC grade improved in some patients.

Predictors of thrombus development and severe SEC on TEE at repeat ablation

Among the patients examined by TEE at repeat ablation, 8 and 6 patients showed SEC grades 3 and 4 , respectively. No patients developed thromboembolic events among those without screening by any imaging modality prior to the repeat ablation. Univariate logistic regression analysis demonstrated that a history of heart failure, LVEF at repeat ablation, history of thrombus detection before the initial ablation, repeat ablation performed $>180$ days, and non-paroxysmal status of atrial arrhythmias after the first session were associated with the development of thrombus and severe SEC. Multivariate analysis showed that repeat ablation $>180$ days (odds ratio $[\mathrm{OR}] 13.0,95 \%$ confidence interval [CI] [1.68-100.9], $\mathrm{p}=0.014$ ), non-paroxysmal atrial arrhythmia after the first session (OR 3.89, 95\% CI [1.36-11.1], p = 0.011), and lower LVEF (OR 0.94, $95 \%$ CI $[0.90-0.99], \mathrm{p}=0.012)$ were independent predictors of thrombus development and severe SEC at repeat ablation (Table 8).

\section{Discussion}

In this large cohort study which ran for over 10 years, the detection of LA thrombus at repeat sessions after initial catheter ablation for AF was very low $(0.8 \%)$ in patients who underwent TEE examination and $0.5 \%$ in the total sample of patients who underwent repeat ablation. The use of imaging modalities at repeat ablation was increased when performed $>180$ days after the first procedure, and all patients who developed LA thrombi were scheduled to undergo repeat ablation $>180$ days after the initial ablation. No patients who did not undergo any imaging evaluation during the repeat session developed adverse events. Repeat ablation >180 days, non-paroxysmal atrial arrhythmia, and lower LVEF were independent predictors of thrombi development and severe SEC at repeat ablation in the multivariate analysis.

LA thrombi are unlikely to develop after initial catheter ablation for AF rather than prior to the first session, given the significantly decreased AF burden and continuous anticoagulant use after ablation. ${ }^{10-12}$ However, little is known about the prevalence of thrombus development and the requirements for imaging evaluation at repeat ablation are unclear. There is only one previous study demonstrating a $0.8 \%$ rate of thrombi detection in 263 patients who underwent repeat TEE within 365 days. ${ }^{13}$ Nonetheless, all patients were prescribed warfarin, a vitamin $\mathrm{K}$ antagonist, in the previous study which was conducted over a long period of time. In contrast, an alternative anticoagulant, DOAC, which is mostly used in AF ablation, has the advantage of quickly promoting and maintaining its anticoagulant effect, which could support the suppression of thrombus development after ablation. Our study is unique in that it evaluated outcomes of using various imaging modalities in patients who received DOACs and warfarin, reflecting a recent trend in clinical practice.

However, despite the presumed safety and efficacy profiles of DOAC, three patients still developed LA thrombi at repeat ablation in our study, which indicates that a reduced risk remains and caution is still warranted at repeat ablation. More importantly, the thrombi developed even in patients with grade 0 SEC in the first session, and there is no guarantee of the absence of thrombi in reference to the examination findings obtained during the first session. Nonetheless, all these patients had a possible reason for the development of thrombi in our study, such as the long period until repeat ablation, inappropriate DOAC use, and decreased cardiac function prior to repeat ablation. In this regard, all patients who developed thrombi were scheduled to undergo repeat ablation $>180$ days after the first procedure, and the frequency of imaging evaluation was significantly increased when the repeat ablation was performed $>180$ days after the first procedure as compared to when performed at [?] 180 days, which suggests that this cutoff duration value should be one of the safety parameters when considering the need for imaging evaluation to detect thrombi before repeat ablation. Moreover, a possible association with the risk of thrombus development in patients with decreased LVEF and non-paroxysmal atrial arrhythmia was mentioned in a previous report. ${ }^{13}$ Although a sub-therapeutic dose of warfarin should be avoided considering its significant relation to thrombus development despite less frequent use of warfarin to date, inappropriate DOAC dose use also increases the 
periprocedural risk of thrombi. ${ }^{13}, 14$ Nonetheless, with an expandable use of DOAC uninterruptedly during ablation, it may be possible to selectively screen patients to identify those at low risk of developing thrombi and those at high risk and who require TEE at repeat ablation. ${ }^{15}$ As long as patients with a low-risk profile continue on oral anticoagulants and undergo early repeat ablation, any imaging modality to determine the presence of LA thrombi may be omitted. Our results could prove useful in deciding whether imaging should be scheduled before repeat ablation according to each patient's clinical profile.

In addition to TEE, contrast-enhanced CT and ICE are useful alternatives to exclude the possibility of LA thrombi, with the hope of obviating the need for TEE before ablation. In a meta-analysis of studies using delayed CT imaging protocols to screen for LA thrombi, the diagnostic accuracy for detection of LAA thrombi was reported to be nearly $100 \% .{ }^{9}$ However, obtaining the CT image some time before the planned ablation day may limit its value, as indicated for Patient 2 in Table 4 . On the other hand, previous data suggested that ICE could be valuable in safely and effectively detecting LA thrombi, whereas the result reflected in large part the variability seen when performing and interpreting the ICE imaging. ${ }^{16-18}$ Moreover, all patients in which thrombi were detected in the present study had D-dimer levels within normal limits before repeat ablation; ${ }^{19}$ therefore, any imaging modality should still be used to screen for thrombi in patients at presumed risk of developing thrombi and those who cannot undergo TEE imaging.

Study limitations

This was a retrospective study conducted in a single center. The decision to plan repeat ablation and the imaging modality of TEE and CT imaging were dependent on the discretion of the attending physician and patient condition. Although we followed medical information and clinical events after the initial ablation in all patients as much as possible, some unknown events and information collected at other facilities could not be obtained from the patients' medical records, resulting in underestimation of the outcomes. Since periprocedural events, especially thromboembolic events, were evaluated as those associated with any symptom or disability, we could not assess asymptomatic embolic events that could be evaluated in a post-procedural head magnetic resonance imaging study. Further large-scale studies evaluating the presence of LA thrombi using various imaging modalities at different time points after ablation are needed.

\section{Conclusions}

The incidence of LA thrombi at repeat ablation for AF was quite low, with fewer imaging examinations being performed at earlier repeat ablation periods. Several significant factors, including later repeat ablation, low LVEF, and non-paroxysmal atrial arrhythmia at repeat ablation, were associated with the risk of developing LA thrombi. Our findings could provide guidance to the clinical decision on whether TEE and CT imaging should be planned before repeat ablation in reasonable patients and could suggest the usefulness of risk stratification to avoid unnecessary screening examinations in certain low-risk populations.

Acknowledgments: none

\section{References}

1. Kuck KH, Brugada J, Furnkranz A, Metzner A, Ouyang F, Chun KR, Elvan A, Arentz T, Bestehorn K, Pocock SJ, Albenque JP, Tondo C: Cryoballoon or Radiofrequency Ablation for Paroxysmal Atrial Fibrillation. N Engl J Med 2016;374:2235-2245.

2. Packer DL, Mark DB, Robb RA, Monahan KH, Bahnson TD, Poole JE, Noseworthy PA, Rosenberg YD, Jeffries N, Mitchell LB, Flaker GC, Pokushalov E, Romanov A, Bunch TJ, Noelker G, Ardashev A, Revishvili A, Wilber DJ, Cappato R, Kuck KH, Hindricks G, Davies DW, Kowey PR, Naccarelli GV, Reiffel JA, Piccini JP, Silverstein AP, Al-Khalidi HR, Lee KL: Effect of Catheter Ablation vs Antiarrhythmic Drug Therapy on Mortality, Stroke, Bleeding, and Cardiac Arrest Among Patients With Atrial Fibrillation: The CABANA Randomized Clinical Trial. JAMA 2019;321:1261-1274.

3. Calkins H, Hindricks G, Cappato R, Kim YH, Saad EB, Aguinaga L, Akar JG, Badhwar V, Brugada J, Camm J, Chen PS, Chen SA, Chung MK, Nielsen JC, Curtis AB, Davies DW, Day JD, d'Avila A, 
de Groot N, Di Biase L, Duytschaever M, Edgerton JR, Ellenbogen KA, Ellinor PT, Ernst S, Fenelon G, Gerstenfeld EP, Haines DE, Haissaguerre M, Helm RH, Hylek E, Jackman WM, Jalife J, Kalman JM, Kautzner J, Kottkamp H, Kuck KH, Kumagai K, Lee R, Lewalter T, Lindsay BD, Macle L, Mansour M, Marchlinski FE, Michaud GF, Nakagawa H, Natale A, Nattel S, Okumura K, Packer D, Pokushalov E, Reynolds MR, Sanders P, Scanavacca M, Schilling R, Tondo C, Tsao HM, Verma A, Wilber DJ, Yamane T: 2017 HRS/EHRA/ECAS/APHRS/SOLAECE expert consensus statement on catheter and surgical ablation of atrial fibrillation. Heart Rhythm 2017;14:e275-e444.

4. Andrade JG, Deyell MW, Verma A, Macle L, Champagne J, Leong-Sit P, Novak P, Badra-Verdu M, Sapp J, Khairy P, Nattel S: Association of Atrial Fibrillation Episode Duration With Arrhythmia Recurrence Following Ablation: A Secondary Analysis of a Randomized Clinical Trial. JAMA network open 2020;3:e208748.

5. Yanagisawa S, Inden Y, Fujii A, Sakamoto Y, Tomomatsu T, Mamiya K, Okamoto H, Shibata R, Murohara T: Prothrombotic Responses After Catheter Ablation for Atrial Fibrillation During Uninterrupted Oral Anticoagulant Agent Administration. JACC Clin Electrophysiol 2019;5:1418-1427.

6. Group JCSJW: Guidelines for Pharmacotherapy of Atrial Fibrillation (JCS 2013). Circ J 2014;78:19972021.

7. Yanagisawa S, Inden Y, Ohguchi S, Nagao T, Fujii A, Tomomatsu T, Mamiya K, Okamoto H, Sakamoto Y, Shibata R, Murohara T: Periprocedural Management of Cardiac Tamponade During Catheter Ablation for AF Under Uninterrupted DOAC and Warfarin. JACC Clin Electrophysiol 2020;6:786-795.

8. Fatkin D, Kelly RP, Feneley MP: Relations between left atrial appendage blood flow velocity, spontaneous echocardiographic contrast and thromboembolic risk in vivo. J Am Coll Cardiol 1994;23:961-969.

9. Romero J, Husain SA, Kelesidis I, Sanz J, Medina HM, Garcia MJ: Detection of left atrial appendage thrombus by cardiac computed tomography in patients with atrial fibrillation: a meta-analysis. Circ Cardiovasc Imaging 2013;6:185-194.

10. Puwanant S, Varr BC, Shrestha K, Hussain SK, Tang WH, Gabriel RS, Wazni OM, Bhargava M, Saliba WI, Thomas JD, Lindsay BD, Klein AL: Role of the CHADS2 score in the evaluation of thromboembolic risk in patients with atrial fibrillation undergoing transesophageal echocardiography before pulmonary vein isolation. J Am Coll Cardiol 2009;54:2032-2039.

11. Wyrembak J, Campbell KB, Steinberg BA, Bahnson TD, Daubert JP, Velazquez EJ, Samad Z, Atwater BD: Incidence and Predictors of Left Atrial Appendage Thrombus in Patients Treated With Nonvitamin K Oral Anticoagulants Versus Warfarin Before Catheter Ablation for Atrial Fibrillation. Am J Cardiol 2017;119:1017-1022.

12. Goldi T, Krisai P, Knecht S, Aeschbacher S, Spies F, Zeljkovic I, Kaufmann BA, Schaer B, Conen D, Reichlin T, Osswald S, Sticherling C, Kuhne M: Prevalence and Management of Atrial Thrombi in Patients With Atrial Fibrillation Before Pulmonary Vein Isolation. JACC: Clinical Electrophysiology 2019;5:14061414 .

13. Smith SA, Binkley PF, Foraker RE, Nagaraja HN, Orsinelli DA: The role of repeat transesophageal echocardiography in patients without atrial thrombus prior to cardioversion or ablation. J Am Soc Echocardiogr 2012;25:1106-1112.

14. Shen X, Li H, Rovang K, Hee T, Holmberg MJ, Mooss AN, Mohiuddin SM: Transesophageal echocardiography before cardioversion of recurrent atrial fibrillation: does absence of previous atrial thrombi preclude the need of a repeat test? Am Heart J 2003;146:741-745.

15. Patel K, Natale A, Yang R, Trivedi C, Romero J, Briceno D, Mohanty S, Alviz I, Natale V, Sanchez J, Della Rocca DG, Tarantino N, Zhang XD, Mohanty P, Horton R, Burkhardt D, Gopinathannair R, Joseph Gallinghouse G, Lakkireddy D, Di Biase L: Is transesophageal echocardiography necessary in patients 
undergoing ablation of atrial fibrillation on an uninterrupted direct oral anticoagulant regimen? Results from a prospective multicenter registry. Heart Rhythm 2020;17:2093-2099.

16. Di Biase L, Briceno DF, Trivedi C, Mohanty S, Gianni C, Burkhardt JD, Mohanty P, Bai R, Gunda S, Horton R, Bailey S, Sanchez JE, Al-Ahmad A, Hranitzky P, Gallinghouse GJ, Reddy YM, Zagrodzky J, Hongo R, Beheiry S, Lakkireddy D, Natale A: Is transesophageal echocardiogram mandatory in patients undergoing ablation of atrial fibrillation with uninterrupted novel oral anticoagulants? Results from a prospective multicenter registry. Heart Rhythm 2016;13:1197-1202.

17. Diab M, Wazni OM, Saliba WI, Tarakji KG, Ballout JA, Hutt E, Rickard J, Baranowski B, Tchou P, Bhargava M, Chung M, Varma N, Martin DO, Dresing T, Callahan T, Cantillon D, Kanj M, Hussein AA: Ablation of Atrial Fibrillation Without Left Atrial Appendage Imaging in Patients Treated With Direct Oral Anticoagulants. Circ Arrhythm Electrophysiol 2020;13:e008301.

18. Anter E, Silverstein J, Tschabrunn CM, Shvilkin A, Haffajee CI, Zimetbaum PJ, Buxton AE, Josephson ME, Gelfand E, Manning WJ: Comparison of intracardiac echocardiography and transesophageal echocardiography for imaging of the right and left atrial appendages. Heart Rhythm 2014;11:1890-1897.

19. Milhem A, Ingrand P, Treguer F, Cesari O, Costa AD, Pavin D, Rivat P, Badenco N, Abbey S, Zannad N, Winum PF, Mansourati J, Maury P, Bader H, Savoure A, Sacher F, Andronache M, Allix-Beguec C, Chillou CD, Anselme F: Exclusion of Intra-Atrial Thrombus Diagnosis Using D-Dimer Assay Before Catheter Ablation of Atrial Fibrillation. JACC: Clinical Electrophysiology 2019;5:223-230.

\section{Figure legends}

Figure 1

Distribution of time to repeat ablation in patients with and without TEE examinations at repeat ablation. TEE, transesophageal echocardiography

Figure 2

Distribution of time to repeat ablation in patients with and without TEE, CT, or ICE examinations at repeat ablation. CT; computed tomography; ICE, intracardiac echocardiography; TEE, transesophageal echocardiography

Figure 3

Prevalence of the evaluation of LA thrombi at different times to repeat ablation using various imaging modalities. CT; computed tomography; ICE, intracardiac echocardiography; LA, left atrium; TEE, transesophageal echocardiography

Figure 4

Clinical characteristics associated with undergoing TEE or any imaging modality including TEE, CT, or ICE at repeat ablation. CI, confidence interval; CT; computed tomography; ICE, intracardiac echocardiography; OR, odds ratio; TEE, transesophageal echocardiography. Other abbreviations as in Table 1.

Figure 5

Change in SEC level from first to repeat ablation in the total sample and in patients who underwent repeat ablation [?]180 days and >180 days after the initial procedure. SEC, spontaneous echocardiography contrast; TEE transesophageal echocardiography. 
Figure 1

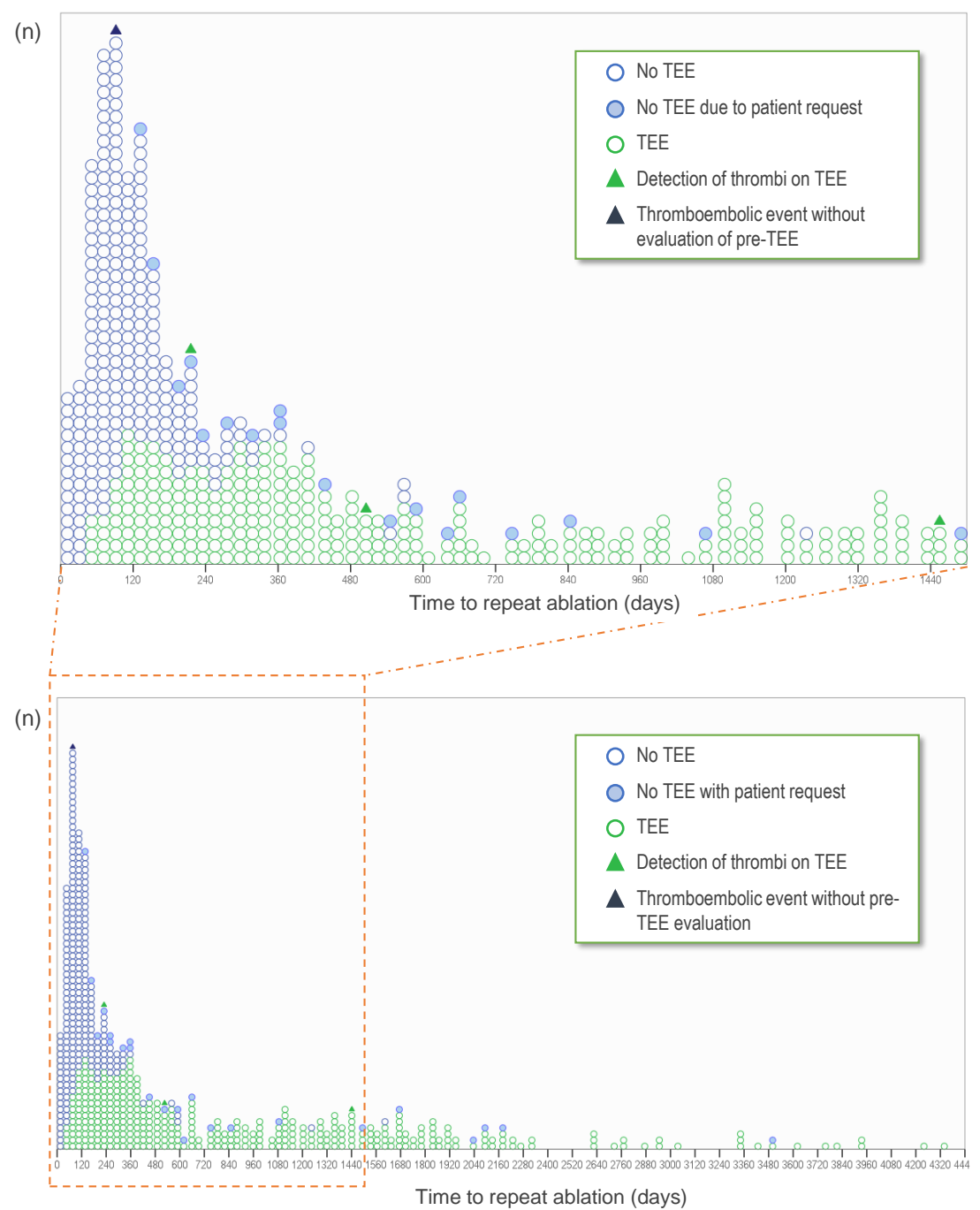


Figure 2

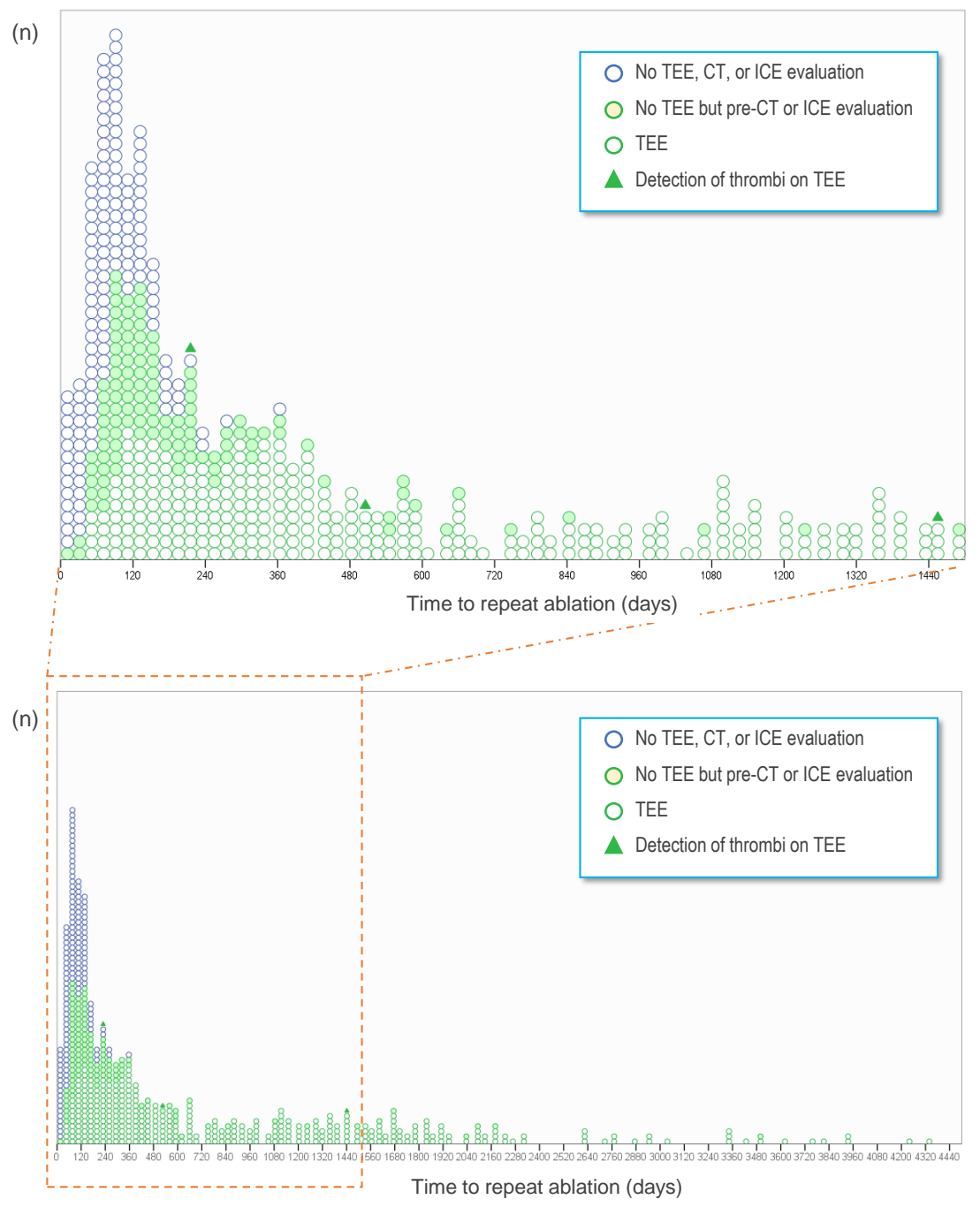


Figure 3

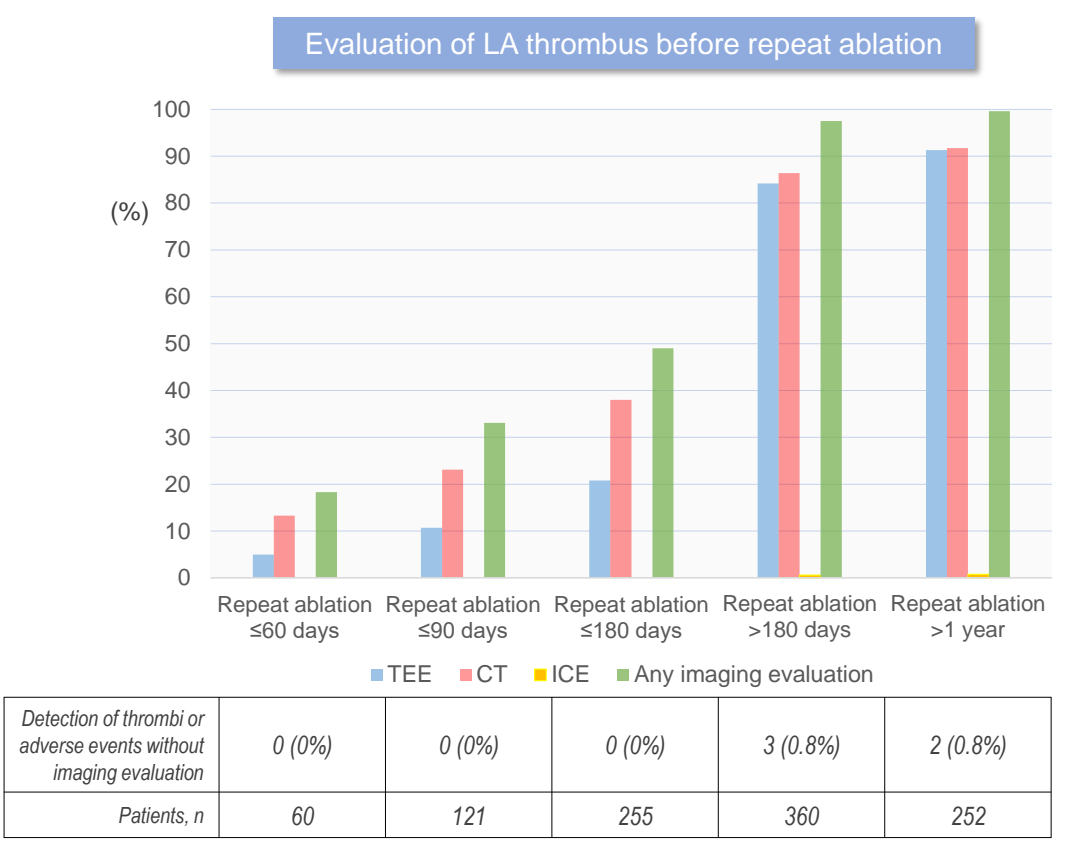


Figure 4

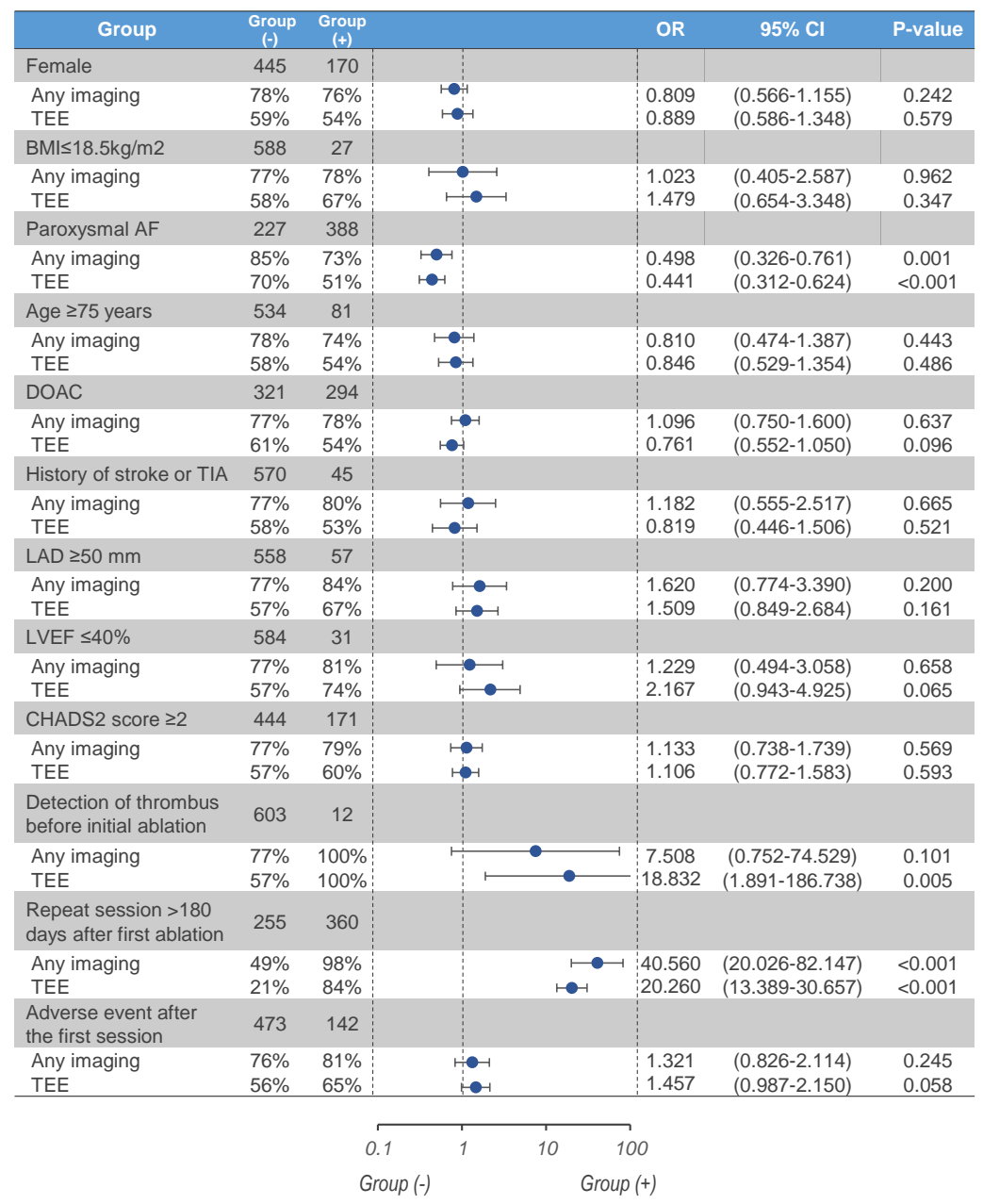


Figure 5
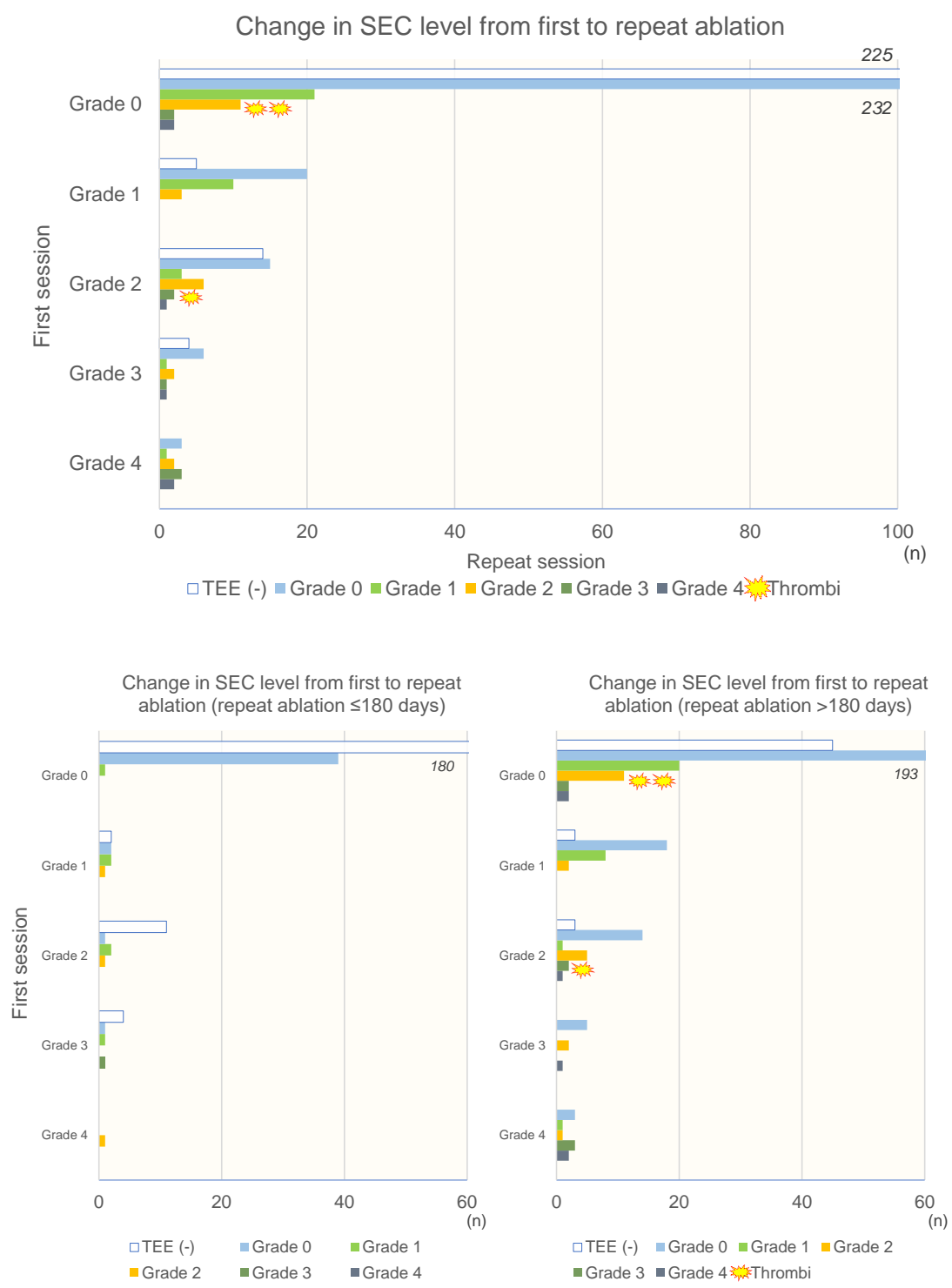

\section{Hosted file}

Table 1.pdf available at https://authorea.com/users/399402/articles/512207-incidenceand-predictors-of-left-atrial-thrombus-development-in-patients-scheduled-for-repeatcatheter-ablation-for-atrial-fibrillation-is-transesophageal-echocardiography-alwaysmandatory

\section{Hosted file}

Table 2.pdf available at https://authorea.com/users/399402/articles/512207-incidenceand-predictors-of-left-atrial-thrombus-development-in-patients-scheduled-for-repeatcatheter-ablation-for-atrial-fibrillation-is-transesophageal-echocardiography-always- 
mandatory

\section{Hosted file}

Table 3.pdf available at https://authorea.com/users/399402/articles/512207-incidenceand-predictors-of-left-atrial-thrombus-development-in-patients-scheduled-for-repeatcatheter-ablation-for-atrial-fibrillation-is-transesophageal-echocardiography-alwaysmandatory

\section{Hosted file}

Table 4.pdf available at https://authorea.com/users/399402/articles/512207-incidenceand-predictors-of-left-atrial-thrombus-development-in-patients-scheduled-for-repeatcatheter-ablation-for-atrial-fibrillation-is-transesophageal-echocardiography-alwaysmandatory

\section{Hosted file}

Table 5.pdf available at https://authorea.com/users/399402/articles/512207-incidenceand-predictors-of-left-atrial-thrombus-development-in-patients-scheduled-for-repeatcatheter-ablation-for-atrial-fibrillation-is-transesophageal-echocardiography-alwaysmandatory

\section{Hosted file}

Table 6.pdf available at https://authorea.com/users/399402/articles/512207-incidenceand-predictors-of-left-atrial-thrombus-development-in-patients-scheduled-for-repeatcatheter-ablation-for-atrial-fibrillation-is-transesophageal-echocardiography-alwaysmandatory

\section{Hosted file}

Table 7.pdf available at https://authorea.com/users/399402/articles/512207-incidenceand-predictors-of-left-atrial-thrombus-development-in-patients-scheduled-for-repeatcatheter-ablation-for-atrial-fibrillation-is-transesophageal-echocardiography-alwaysmandatory

\section{Hosted file}

Table 8.pdf available at https://authorea.com/users/399402/articles/512207-incidenceand-predictors-of-left-atrial-thrombus-development-in-patients-scheduled-for-repeatcatheter-ablation-for-atrial-fibrillation-is-transesophageal-echocardiography-alwaysmandatory 